\title{
Armonie e dissonanze sul pentagramma dei pronomi
}

\author{
Sandro Spinsanti \\ Direttore Istituto Giano per le Medical Humanities, Roma - Italy
}

Che musica è prodotta dalla pandemia di Covid-19? Proviamo a immaginarla distribuendo i nostri comportamenti sul pentagramma dei pronomi personali: quelle parolette che costituiscono il codice genetico da cui si sviluppa la vita: personale e sociale. Cominciando, ovviamente, dall'IO. II più fondamentale dei pronomi, senza il quale non c'è consapevolezza; potenzialmente anche il più odioso. Quando canta da solista, può generare stridule dissonanze. Soprattutto se è il pilastro centrale di quella religione che si chiama egolatria. Certo, è la colonna vertebrale dell'assunzione di responsabilità, quella che si esprime in un "IO mi prendo la responsabilità" (di decisioni necessarie, in un contesto di autorità balbettanti e contraddittorie, che hanno valso l'immagine di "scimmie al volante" a politici, amministratori e autoaccreditatisi esperti che ci hanno guidato in tempo di pandemia); "IO sono responsabile della protezione dei più fragili". E adotta questi comportamenti in rapporto con il TU. "Mi metto la mascherina, mantengo i distanziamenti prescritti perché la TUA salute mi sta a cuore". Ma I'IO, soprano femminile, tenore maschile, controtenore ..., può anche intonare un "Di te non m'importa niente". Può rivendicare una resistenza a oltranza contro ogni limitazione di libertà, può innalzarsi nell'acuto del negazionista: "Tutte fandonie per paralizzare i cittadini e renderli succubi di una dittatura soft; IO so come stanno veramente le cose". Oppure, di fronte al TU, la mascherina può diventare l'espressione della diffidenza sistematica: "Ognuno è un potenziale untore e IO proteggo me stesso: stammi lontano - TU!".

Diversi 10 si possono aggregare in NOI. Quel NOI che diventa un canto corale: "NOI condomini, NOI infermieri, NOI medici ...". E trovare delle sintesi che, nella normalità, non era dato registrare, come: “Collega, mi aiuti?", perché la scienza annaspa nelle spiegazioni; IO sono al limite delle mie forze e, se ci uniamo, forse possiamo ottenere di più ...; è l'inusuale rubrica che ci è dato di trovare nel sito Internet, frequentato

Received: January 2, 2021

Accepted: January 14, 2021

Published online: February 18, 2021 da 100.000 medici, dal quale è stato tratto il volume: Emozioni virali (II Pensiero Scientifico). II NOI crea le migliori armonie quando tutte le forze, di solito antagoniste, si alleano per un obiettivo comune, per esempio, lo sforzo sinergico e collaborativo dei ricercatori e delle aziende che costituiscono Big Pharma per la creazione di vaccini. Altro motivo felice del canto comune: "NOI ci fidiamo del vaccino; la nostra libertà individuale confluisce in una comunità solidale. Siamo, infatti, diventati consapevoli che ci si salva solo insieme: se non è collettiva, la salvezza è impossibile".

Anche il pronome NOI produce possibili dissonanze. Succede, per esempio, quando il NOI diventa un'aggregazione battagliera contro il VOI. Rivendicando "Prima i nostri" o "Prima quelli impegnati nella produzione, poi quelli che l'economia del Paese trascina a rimorchio". II NOI può diventare stridulo soprattutto quando, nella palude delle vittime, spuntano ESSI: quelli dei quali possiamo fare a meno, perché troppo anziani (dare la priorità, nelle terapie intensive, ai pazienti con maggiore speranza di vita ...), perché non più produttivi. Nessuno osa esprimere un cinico senso di liberazione per aver scaricato dalla società un buon numero di pesi morti, ma, con un po' di applicazione, nella musica dominante il motivo nascosto può essere individuato. Perché, quando la zattera galleggia a stento e rischia di portare tutti a fondo, bisognerà pur alleggerire il carico, senza pietismi ...

Nel canto emerge anche il diverso valore di LUI/LEI, a seconda che abbia o meno un legame profondo con I'IO e con il NOI. Perché questa terza persona può andare a finire nella discarica di ESSI: quelli che né gli affetti né gli interessi mantengono sistematicamente legati all'IO e al NOI. Massimamente quando fossero gettati sulle nostre rive dalla risacca dell'immigrazione.

Per una società diversa abbiamo bisogno che su tutti i pronomi i canti si armonizzino, senza stonature. IO proteggo dal virus ME e TE; insieme, NOI proteggiamo LUI/LEI, che è il più debole; ci diamo una mano per tirarci fuori dalla crisi economica in cui siamo precipitati: NOI e VOI insieme. ESSI, i più lontani dai nostri interessi, ci faranno scoprire una società nuova. Il titolo del canto? Potrebbe essere: "FRATELLI TUTTI".

Indirizzo per la corrispondenza:

Sandro Spinsanti

Istituto Giano

Via Stazzo Quadro 7

00060 Riano, Roma - Italy

sandro.spinsanti@gmail.com 